\title{
Skull bone marrow injury caused by MR-guided focused ultrasound for cerebral functional procedures
}

\author{
Michael L. Schwartz, MD, MSc, ${ }^{1,2}$ Robert Yeung, MD, ${ }^{2,3}$ Yuexi Huang, PhD, ${ }^{4}$ \\ Nir Lipsman, MD, PhD, ${ }^{1,2}$ Vibhor Krishna, MD, SM, ${ }^{5}$ Jennifer D. Jain, MD, ${ }^{2}$ \\ Martin G. Chapman, BM, ${ }^{2,6}$ Andres M. Lozano, MD, PhD, ${ }^{1,7}$ and Kullervo Hynynen, PhD ${ }^{4,8,9}$

\begin{abstract}
${ }^{1}$ Division of Neurosurgery and Departments of ${ }^{3}$ Medical Imaging and ${ }^{6}$ Anaesthesia, ${ }^{8}$ Medical Biophysics, and ${ }^{9}$ nstitute of Biomaterials and Biomedical Engineering, University of Toronto; ${ }^{2}$ Sunnybrook Health Sciences Centre; ${ }^{4}$ Physical Sciences Platform, Sunnybrook Research Institute; and ${ }^{7}$ Toronto Western Hospital, Toronto, Ontario, Canada; and ${ }^{5}$ Department of Neurosurgery, The Ohio State University, Columbus, Ohio
\end{abstract}

OBJECTIVE One patient for whom an MR-guided focused ultrasound (MRgFUS) pallidotomy was attempted was discovered to have multiple new skull lesions with the appearance of infarcts on the MRI scan 3 months after his attempted treatment. The authors conducted a retrospective review of the first 30 patients treated with MRgFUS to determine the incidence of skull lesions in patients undergoing these procedures and to consider possible causes.

METHODS A retrospective review of the MRI scans of the first 30 patients, 1 attempted pallidotomy and 29 ventral intermediate nucleus thalamotomies, was conducted. The correlation of the mean skull density ratio (SDR) and the maximum energy applied in the production or attempted production of a brain lesion was examined.

RESULTS Of 30 patients treated with MRgFUS for movement disorders, 7 were found to have new skull lesions that were not present prior to treatment and not visible on the posttreatment day $1 \mathrm{MRI}$ scan. Discomfort was reported at the time of treatment by some patients with and without skull lesions. All patients with skull lesions were completely asymptomatic. There was no correlation between the mean SDR and the presence or absence of skull lesions, but the maximum energy applied with the Exablate system was significantly greater in patients with skull lesions than in those without.

CONCLUSIONS It is known that local skull density, thickness, and SDR vary from location to location. Sufficient energy transfer resulting in local heating sufficient to produce a bone lesion may occur in regions of low SDR. A correlation of lesion location and local skull properties should be made in future studies.

https://thejns.org/doi/abs/10.3171/2017.11.JNS17968

KEYWORDS focused ultrasound; thalamotomy; skull lesions; pallidotomy; transcranial ultrasound; functional neurosurgery

$\mathrm{F}$ OCUSED ultrasound (FUS) offers a minimally invasive method of tissue ablation that was first reported in $1942 .{ }^{9}$ At first, FUS beams were guided by radiography and bony landmarks, but in 1993, Hynynen and colleagues described the use of MRI for guidance. ${ }^{6}$ MRI offers the advantage of 3D targeting and, in its current application, permits real-time imaging and thermography. Accurate placement of the lesion and control of the maximum temperature generated enhance the safety of treatments performed using this modality. Furthermore, for intracranial treatments, a phased array of (currently) up to 1024 ultrasound transducers usually allows a tightly focused hot spot, even though a considerable proportion of the energy is absorbed by the skull. ${ }^{3}$

Ultrasound energy loss in the skull bone depends on the skull thickness and density, and it has been shown by computer simulations that uniform skull surface intensity can result in local hot spots in the skull. ${ }^{3}$ Similarly, early experiments in nonhuman primates showed local thermal damage on the brain surface presumably as a result of local bone heating.? One factor that may correlate with the deposition of thermal energy in the skull is reported to 


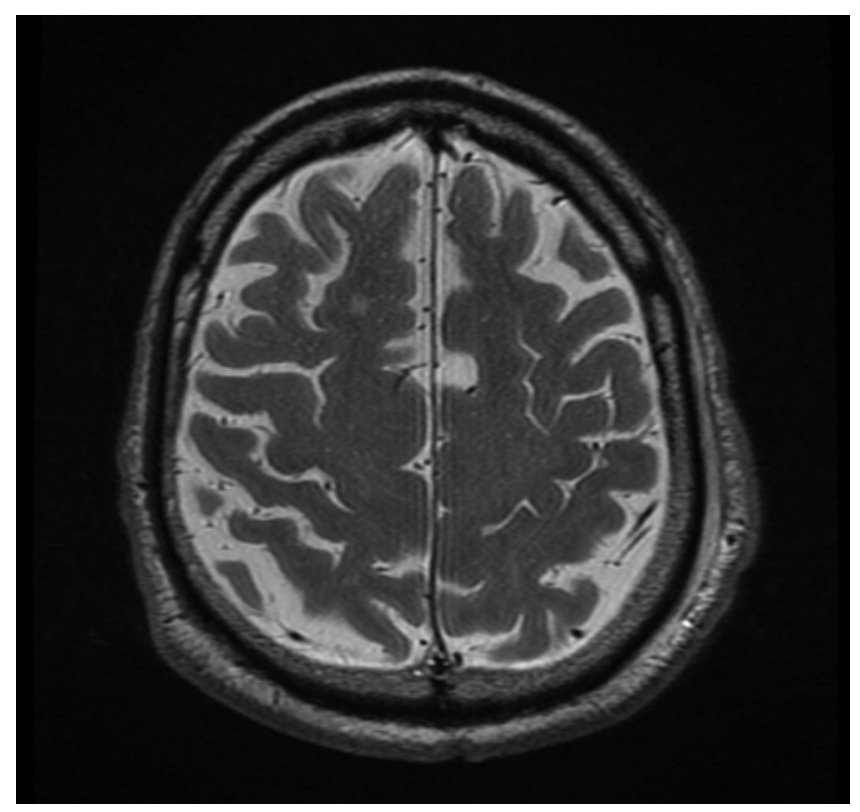

FIG. 1. Index case. This patient underwent sonication increasing to a maximum power of $1100 \mathrm{~W}$ for a duration of 31 seconds. During the procedure, the scalp and skull were constantly cooled with flowing degassed water at $13^{\circ} \mathrm{C}$. Despite this sonication, the target locus in the internal globus pallidus reached only $48^{\circ} \mathrm{C}$. No brain or skull lesions are visible on this day 1 posttreatment axial MRI scan.

be the skull density ratio (SDR). ${ }^{2}$ The SDR is calculated in small sections over the skull as the ratio in Hounsfield units for marrow and cortical bone. The individual section values are averaged, and a global measurement is calculated for each patient. Patients were excluded from the recent prospective randomized trial if their mean SDR was less than $0.4 .^{5}$ In this study, we analyzed patients whom we treated with FUS with the intent to produce focal ablation either for essential tremor ${ }^{8}$ or for Parkinson's disease. Our objective was to determine the incidence of skull lesions in all patients undergoing MR-guided FUS (MRgFUS) procedures and to consider possible causes.

\section{Methods}

A retrospective review of the MRI scans of the first 30 patients who underwent MRgFUS (1 attempted pallidotomy and 29 ventral intermediate nucleus thalamotomies) was conducted. Details of the treatments are provided in previous studies. ${ }^{4,8}$ The correlation of the mean SDR and the maximum energy applied in the production or attempted production of a brain lesion was examined.

\section{Results \\ Index Case}

This 80-year-old right-handed man had L-dopa-induced dyskinesia. The patient's mean SDR was measured at 0.48 . A pallidotomy was attempted in January 2015. A total of 18 sonications with power and time of sonication incrementally increased to a maximum of $34.1 \mathrm{~kJ}$ (1100 $\mathrm{W}$ for a duration of 31 seconds) raised the temperature at

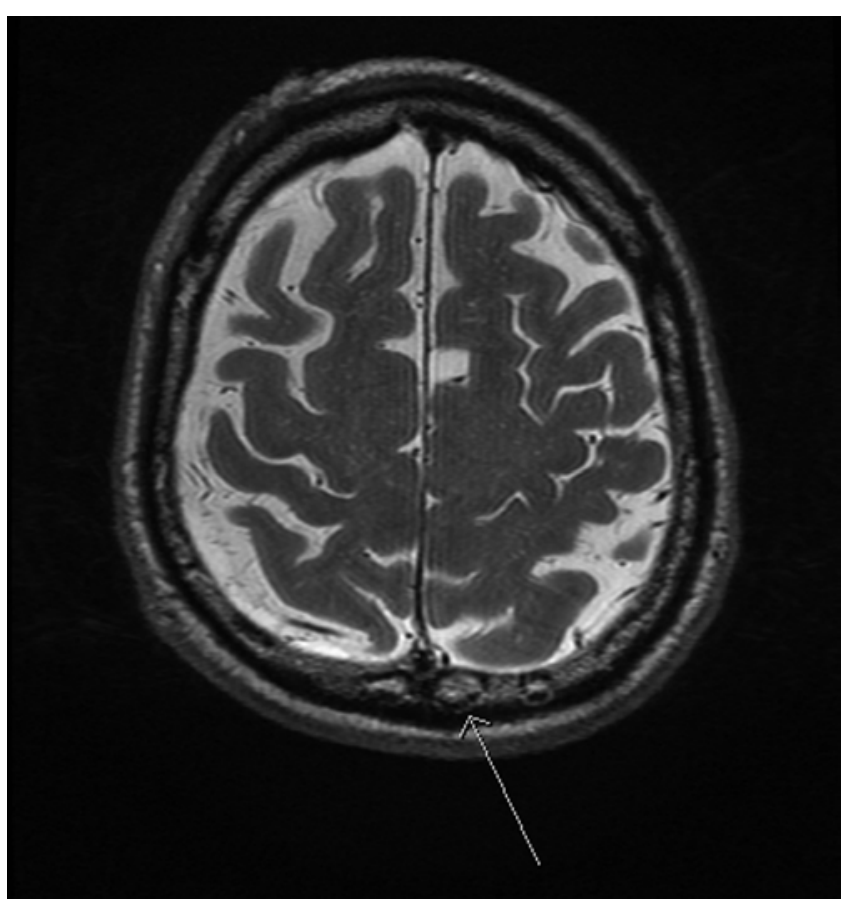

FIG. 2. Axial MRI scan obtained at 3 months posttreatment, showing ovoid lesions throughout the calvaria indicated by the arrow, which were new since the pretreatment MRI scan. Their appearance resembles that of bone infarcts.

the internal globus pallidus target to only $48^{\circ} \mathrm{C}$. Despite analgesia administered during the procedure, the patient complained of pain. A T2-weighted MRI scan was obtained, and no brain lesion was demonstrated. Immediately following the procedure, there was no change in his clinical examination findings. The following morning, the patient was reexamined and a complete MRI study was obtained (Fig. 1). There was a very slight improvement in his right-sided dyskinesia and no change in his MRI scan from the baseline images obtained prior to treatment. As part of our protocol, he was reexamined at 1 month and at 3 months, and an MRI scan was obtained at each visit. At the 1-month examination, there was no treatment effect evident and no bony lesions in the skull. At 3 months, there was no treatment effect, but there were multiple ovoid-shaped areas of signal change with low T1/T2 rims scattered bilaterally throughout the calvaria (Fig. 2), new since the 1-month scan. Their appearance was that of bone infarcts. Another MRI scan was obtained, 8 months after treatment (Fig. 3). The bone lesions were unchanged. The patient was last seen in February 2016. At no time subsequent to his treatment did he complain of or report skull pain or tenderness. His overall condition had declined significantly, and he was subsequently unable to return for further clinical examination or imaging.

\section{Review of the First 30 Patients Treated With MRgFUS}

Of 30 patients treated for movement disorders with MRgFUS, 7 patients were found to have multiple new skull lesions that were not present prior to treatment and 


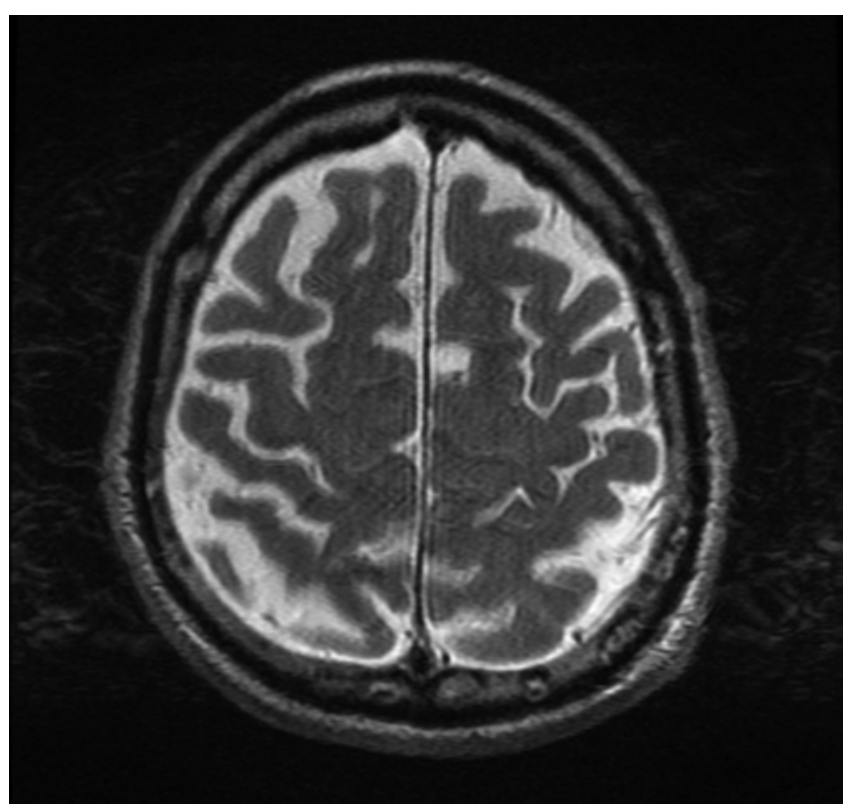

FIG. 3. Axial MRI scan obtained 8 months posttreatment, showing no change in the appearance of the skull lesions.

not visible on the posttreatment scans obtained prior to 3 months posttreatment. Discomfort was reported at the time of treatment by some patients with and without skull lesions. All patients with skull lesions were completely asymptomatic. There were no MRI changes on the brain surface or in the overlying muscle or skin.

One factor that influences the deposition of thermal energy in the skull is reported to be the SDR. ${ }^{2}$ The SDR is calculated in small sections over the skull as the ratio in Hounsfield units for marrow and cortical bone. The individual section values are averaged, and a global measurement is calculated for each patient. Patients were excluded from the recent prospective randomized trial if their mean SDR was less than $0.4 .^{5}$

An attempt to correlate maximum energy and mean SDR with the presence or absence of lesions was made by plotting maximum energy versus SDR and the presence or absence of lesions (Fig. 4). The mean maximum energy for patients with skull lesions was $25.8 \mathrm{~kJ}$. The mean maximum energy for patients without lesions was $15.5 \mathrm{~kJ}$. The difference between these values is statistically significant ( $p=0.00694$, Wilcoxon Mann-Whitney U-test). In Fig. 4, there is a visible separation between patients with skull lesions (red squares) and patients without lesions (green diamonds) with respect to maximum energy, but not for SDR. In our experience, the highest maximum energy treatments are more likely to be associated with skull lesions than lower maximum energy treatments, but there is no correlation between mean SDR and the presence or absence of skull lesions.

\section{Discussion}

The MRI scans obtained 3 months after sonications showed that approximately $20 \%$ of our patients had sig-

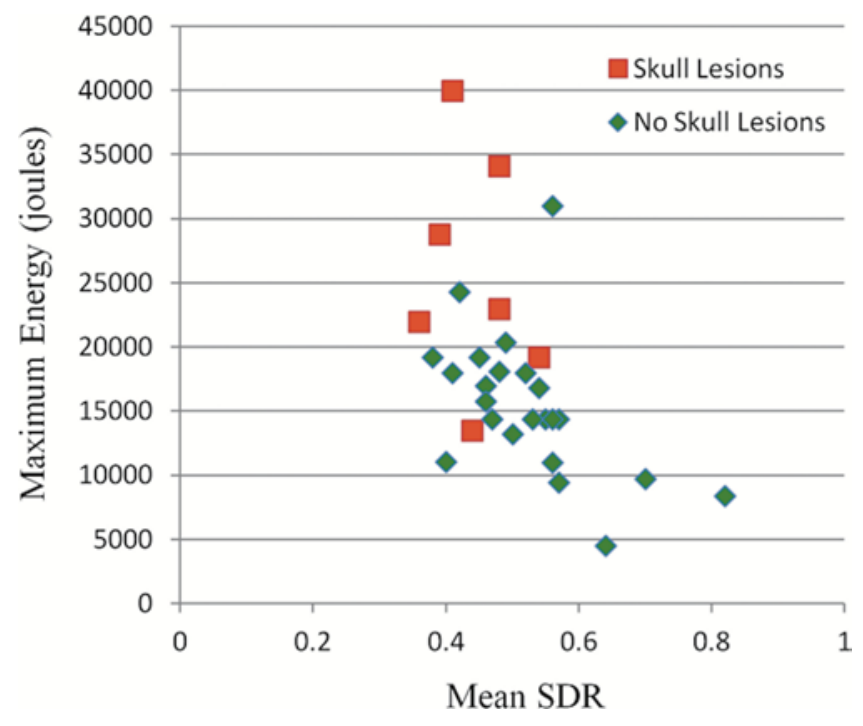

FIG. 4. The mean maximum energy for patients with skull lesions was $25.8 \mathrm{~kJ}$. The mean maximum energy for patients without lesions was $15.5 \mathrm{~kJ}$.

nal intensity changes indicating potential tissue damage. Simulation studies have indicated that local hot spots could be induced inside the skull bone. ${ }^{3}$ Similarly, in preclinical testing in monkeys of the MRgFUS methodology for brain applications in humans, skull heating was significant and sufficient to cause bone and underlying brain lesions. ${ }^{7}$ In this study, only bone abnormalities were detected without changes in the soft tissues on the skull surface.

MRgFUS is used for treatment of osseous lesions, such as bone metastases and osteoid osteomas..$^{10}$ Target temperatures of approximately $60^{\circ} \mathrm{C}$ are sufficient to cause lesions for therapeutic effect. In Fig. 5, the bone temperature at the cursor is sufficient to cause a marrow lesion, but we cannot say that there was, in fact, a lesion at that exact location. The baseline temperature is reduced by cooling the skin to $13^{\circ} \mathrm{C},{ }^{3}$ and thus the peak temperature is likely to be below thermal damage threshold in the tissue on the bone surface. This could explain the lack of soft-tissue lesions in the skin or muscle over the bone.

We did not systematically measure bone heating, but temperatures on the bone surface may be sampled in this way. Local temperatures are observed to vary from point to point. In Fig. 5, the maximum bone surface temperature was $65^{\circ} \mathrm{C}$. In determining whether the actual temperature increase at the target point in the brain corresponds to the expected focal point of the active ultrasound beams, axial, coronal, and sagittal sections are obtained through the focal point only, and a systematic survey of bone temperature is not obtained.

Other factors that may influence skull absorption of energy are beam angle and local skull features. Unfortunately, we were unable to definitively correlate beam angles or local skull features visible on CT with the location of skull lesions. There were no obvious instances of pretreatment abnormalities on CT scans in the vicinity of posttreatment bone lesions identified on MRI scans. 


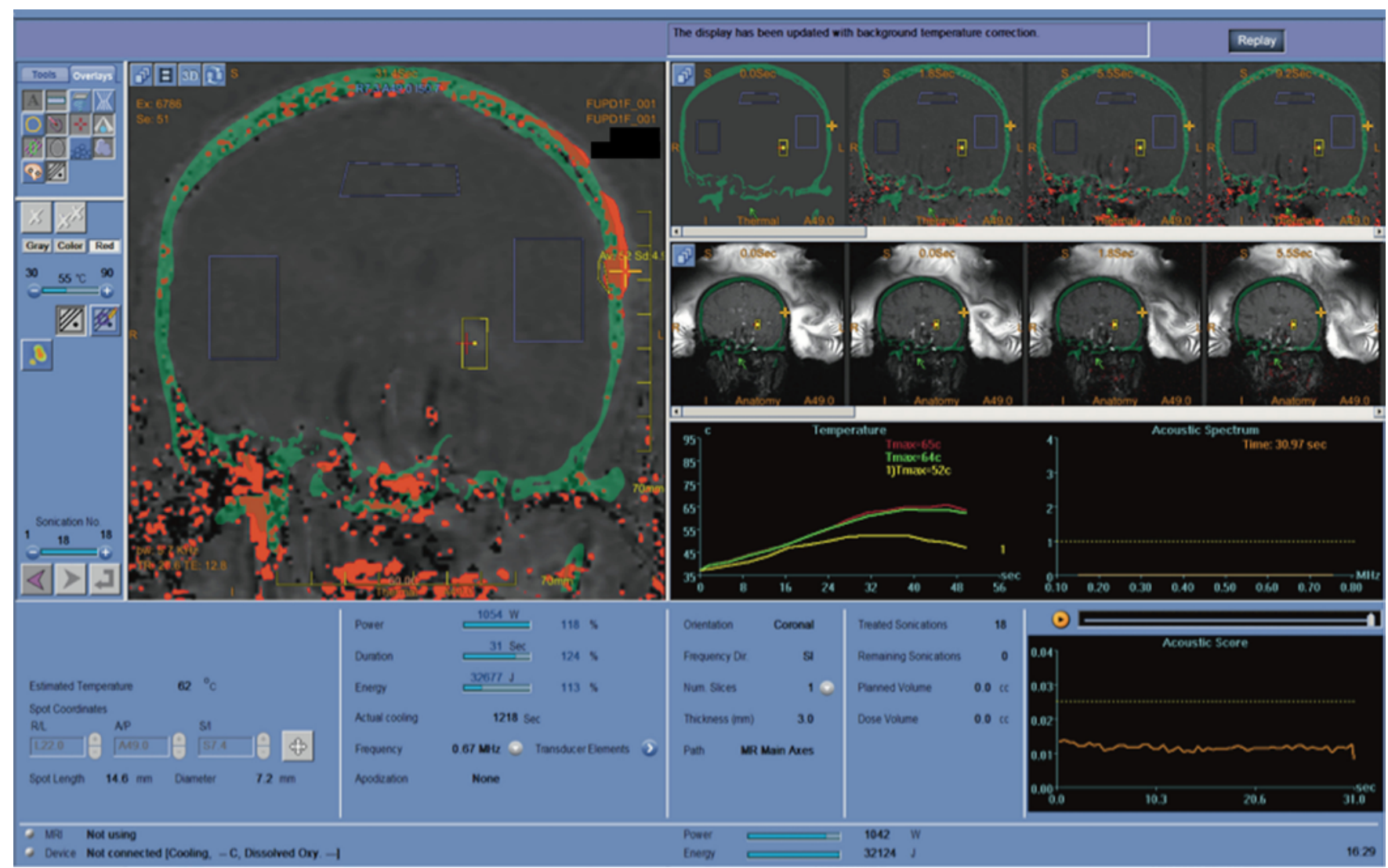

FIG. 5. Coronal temperature map through the target point in the left ventral intermediate nucleus. At the cursor over the left parietal bone, the temperature reaches a maximum of $65^{\circ} \mathrm{C}$ when a baseline temperature of $37^{\circ} \mathrm{C}$ is used. The baseline temperature, however, is reduced by the skin cooling of $13^{\circ} \mathrm{C}$, and thus the peak temperature is likely to be below the threshold of thermal damage in the tissue on the bone surface.

The number of active elements (beams of ultrasound) was considered as a factor in the production of skull lesions. The number of active elements is automatically reduced as power is increased to avoid excessive heating of intracranial lesions visible on the pretreatment CT scan and marked as "no-pass" regions. For patients with skull lesions, the mean number of active elements at maximum power was 802, and for those without lesions, it was 825.8. This difference is not significant $(\mathrm{p}=0.271$, Wilcoxon Mann-Whitney U-test).

In addition to the number of active elements, the skull area available for sonication is measured for each treatment and the energy per square centimeter may be calculated. The skull area available for sonication was 299.6 $\mathrm{cm}^{2}$ in those with lesions, and in those without lesions, the available area was $305.9 \mathrm{~cm}^{2}$, not a significant difference ( $p=0.944$, Wilcoxon Mann-Whitney U-test). As noted above, the difference in maximum energy between those with lesions and those without is significant $(\mathrm{p}=$ 0.00694 ), and as a consequence the energy per square centimeter is significant as well $(p=0.0048)$.

Because all patients with brain lesions were asymptomatic, we did not alter our follow-up MRI schedule for the 6 remaining essential tremor patients with bone lesions and do not have data regarding bone healing. The first patient with bone lesions has undergone follow-up for 63 months. The last patient included in this series has undergone follow-up for 19 months.

In experimental animals subjected to focal bone heating generated by MRgFUS, bone remodeling and healing does occur. In rat femurs, there is evidence of bone healing at 31 days after production of a bone lesion; ${ }^{12}$ in rabbit femurs, there is new bone and cartilage formation adjacent to the necrotic bone at 28 days ${ }^{11}$ and in swine femurs, subtle evidence of new ossification is present at 3 weeks and robust bone healing at 6 weeks. ${ }^{1}$

In our index patient, the skull lesions visible at 3 months were still present at 8 months.

\section{Conclusions}

We were unable to definitively correlate local features visible on the original pretreatment CT scan with the location of the bone lesions that developed and were visible on the 3-month posttreatment MRI scan. We have determined, so far, that the maximum energy applied to produce or attempt to produce a brain lesion does correlate with the production of bone lesions. A prospective study to identify which local features predispose to the development of bone lesions should now be done. 


\section{References}

1. Bucknor MD, Rieke V, Seo Y, Horvai AE, Hawkins RA, Majumdar S, et al: Bone remodeling after MR imaging-guided high-intensity focused ultrasound ablation: evaluation with MR imaging, CT, $\mathrm{Na}^{18} \mathrm{~F}-\mathrm{PET}$, and histopathologic examination in a swine model. Radiology 274:387-394, 2015

2. Chang WS, Jung HH, Zadicario E, Rachmilevitch I, Tlusty T, Vitek S, et al: Factors associated with successful magnetic resonance-guided focused ultrasound treatment: efficiency of acoustic energy delivery through the skull. J Neurosurg 124:411-416, 2016

3. Connor CW, Hynynen K: Patterns of thermal deposition in the skull during transcranial focused ultrasound surgery.

IEEE Trans Biomed Eng 51:1693-1706, 2004

4. Elias WJ, Huss D, Voss T, Loomba J, Khaled M, Zadicario E, et al: A pilot study of focused ultrasound thalamotomy for essential tremor. N Engl J Med 369:640-648, 2013

5. Elias WJ, Lipsman N, Ondo WG, Ghanouni P, Kim YG, Lee $\mathrm{W}$, et al: A randomized trial of focused ultrasound thalamotomy for essential tremor. N Engl J Med 375:730-739, 2016

6. Hynynen K, Damianou C, Darkazanli A, Unger E, Schenck JF: The feasibility of using MRI to monitor and guide noninvasive ultrasound surgery. Ultrasound Med Biol 19:91-92, 1993

7. Hynynen K, McDannold N, Clement G, Jolesz FA, Zadicario E, Killiany R, et al: Pre-clinical testing of a phased array ultrasound system for MRI-guided noninvasive surgery of the brain - a primate study. Eur J Radiol 59:149-156, 2006

8. Lipsman N, Schwartz ML, Huang Y, Lee L, Sankar T, Chapman M, et al: MR-guided focused ultrasound thalamotomy for essential tremor: a proof-of-concept study. Lancet Neurol 12:462-468, 2013

9. Lynn JG, Zwemer RL, Chick AJ, Miller AE: A new method for the generation and use of focused ultrasound in experimental biology. J Gen Physiol 26:179-193, 1942

10. Ozhinsky E, Han M, Bucknor M, Krug R, Rieke V: T2-based temperature monitoring in bone marrow for MR-guided focused ultrasound. J Ther Ultrasound 4:26, 2016

11. Smith NB, Temkin JM, Shapiro F, Hynynen K: Thermal effects of focused ultrasound energy on bone tissue. Ultrasound Med Biol 27:1427-1433, 2001

12. Yeo SY, Arias Moreno AJ, van Rietbergen B, Ter Hoeve ND, van Diest PJ, Grüll H: Effects of magnetic resonance-guided high-intensity focused ultrasound ablation on bone mechanical properties and modeling. J Ther Ultrasound 3:13, 2015

\section{Disclosures}

Dr. Lozano: ownership in Functional Neuromodulation; and consultant for Medtronic, St. Jude, and Boston Scientific. Dr. Hynynen: patent holder with InSightec.

The care of these patients was supported by InSightec Ltd., Tirat Carmel, Israel, and the Focused Ultrasound Foundation, Charlottesville, Virginia.

\section{Author Contributions}

Conception and design: Schwartz. Acquisition of data: Schwartz, Yeung, Huang, Lipsman, Hynynen. Analysis and interpretation of data: Schwartz, Yeung, Huang, Hynynen. Drafting the article: Schwartz. Critically revising the article: Schwartz, Krishna, Chapman, Lozano, Hynynen. Reviewed submitted version of manuscript: all authors. Approved the final version of the manuscript on behalf of all authors: Schwartz. Statistical analysis: Schwartz. Administrative/technical/material support: Krishna, Hynynen.

\section{Supplemental Information Previous Presentations}

This work was previously presented in part at the 16th International Symposium on Therapeutic Ultrasound, Tel Aviv, Israel, March 14-16, 2016.

\section{Correspondence}

Michael L. Schwartz: Sunnybrook Health Sciences Centre, Toronto, ON, Canada.m.schwartz@utoronto.ca. 